\title{
Methotrexate and theaflavin-3,3' di gallate synergistically restore the balance between apoptosis and autophagy in synovial fibroblast of RA: An ex vivo approach with cultured human RA FLS
}

\section{Sanchaita Misra}

Institute of Postgraduate Medical Education and Research

Aniruddha Bagchi

Institute of Postgraduate Medical Education and Research

Avik Sarkar

Institute of Postgraduate Medical Education and Research

Sougata Niyogi

Indian Institute of Chemical Biology CSIR

Dipanjan Bhattacharjee

Institute of Postgraduate Medical Education and Research

\section{Sulagna Chatterjee}

Institute of Postgraduate Medical Education and Research

Sumantro Mondal

Institute of Postgraduate Medical Education and Research

Arghya Chattopadhyay

Institute of Postgraduate Medical Education and Research

Ayindrila Saha

Institute of Postgraduate Medical Education and Research

\section{Sudipta Chatterjee}

Institute of Postgraduate Medical Education and Research

\section{Pradyot Sinhamahapatra}

Institute of Postgraduate Medical Education and Research

Partha Chakrabarti

Indian Institute of Chemical Biology CSIR

\section{Mitali Chatterjee}

Institute of Postgraduate Medical Education and Research

Alakendu Ghosh ( $\sim$ prof.dr.alakendughosh@gmail.com )

Institute of Postgraduate Medical Education and Research https://orcid.org/0000-0003-2956-9703 


\section{Research Article}

Keywords: Rheumatoid arthritis, Fibroblast like synoviocytes, hypoxia, angiogenesis, Endoplasmic reticulum stress, Apoptosis/Autophagy imbalance, TF3, MTX

Posted Date: March 12th, 2021

DOI: https://doi.org/10.21203/rs.3.rs-282810/v1

License: (c) (i) This work is licensed under a Creative Commons Attribution 4.0 International License. Read Full License

Version of Record: A version of this preprint was published at Inflammopharmacology on August 5th, 2021. See the published version at https://doi.org/10.1007/s10787-021-00857-0. 


\section{Abstract}

Background: Rheumatoid arthritis (RA) is characterized by inflammation mediated angiogenesis in synovial tissue, leading to apoptotic retardation and enhanced cell survival in synovial fibroblasts. Methotrexate (MTX) can reduce selective pro-inflammatory cytokines but unable to restore disrupted homeostasis between autophagy and apoptosis in $f d-F L S$.

Objective: To evaluate the effect of black tea compound TF3 along with MTX upon fluid derived ( $f d$ )-FLS to induce apoptosis and inhibit autophagy through ER stress-mediated pathways.

Methods: FLS sourced from synovial fluid (SF) of patients with RA $(n=11)$ and osteoarthritis $(O A)(n=10)$ were cultured following treatment with MTX/TF3 or in combination and underlying mechanisms were investigated. Extracellular inflammatory markers like CRP and cytokines (TNF-a, LL-6), angiogenic markers (VEGF, ANG-1) were quantified by ELISA. Cell viability of cultured $f d$-FLS was determined by MTT assay. $f d$-FLS treated with MTX/TF3 or combination of MTX $(125 \mathrm{nM})$ and TF3 $(10 \mu \mathrm{M})$, followed by apoptosis measurement by flow cytometry. ER stress associated markers were quantified by RT-PCR (IRE1A and spliced-XBP-1) and immunoblotting (Grp78, Hsp70, CHOP, HIF1-a). Apoptotic (Bcl-2, Bax, and Caspases) and autophagic proteins (Beclin1, LC3b and p62) were quantified by immunoblot study.

Results: MTX and TF3 both in single doses $\left(\mathrm{IC}_{25}\right)$ could down-regulate the levels of pro-inflammatory and angiogenic markers. Combination treatment modulated ER stress response and blocked the autophagmosomal proteins in $f d-F L S$ and induced apoptosis.

Conclusion: Disruption in homeostasis between apoptosis and autophagy might be an underlying phenomenon in the progression of pathophysiology in $f d$-FLS. The combined administration of MTX and TF3 successfully balanced the homeostasis by inducing apoptosis.

\section{Introduction}

RA, is systemic, chronic, an autoimmune disorder, mainly associated with inflammation and pain in peripheral joints, which leads to swelling in synovial cell lining as well as pannus formation, termed as 'synovitis' (Guo et al. 2018). Infiltration of synovial fluid-derived fibroblast cells ( $f d-F L S$ ) disrupts the synovium lining that leads to the perpetuation of disease pathogenesis and progression (Stebulis et al. 2005). Within the synovium, increased pro-inflammatory cytokine milieu (TNF-a, IL-6) induces cell proliferation leading to depletion of oxygen $\left(\mathrm{O}_{2}\right)$ and facilitates hypoxic microenvironment. This hypoxic stress eventually triggers the expressions of HIF-1a, VEGF, and angiogenic factors like ANG-1 (Elshabrawy et al. 2015; Quinonez-Flores et al. 2016).

In the endoplasmic reticulum (ER), integral homeostasis is maintained by intricately coordinated adaptive interactions between unfolded protein responses (UPRs) and ER-associated degradations (ERAD) of accumulated misfolded proteins (Park et al. 2014). Imbalance in homeostasis within ER increases the rate of UPR, subsequently ER stress (Guzel et al. 2017). GRP78, a well-recognized molecular chaperon, is 
known to be activated during ER stress and consequently binds to misfolded proteins (Yoo et al. 2012). It has been previously reported that hypoxic stress induced proteins can stimulate ER stress responses and their respective downstream signaling proteins in $f d-F L S$ (Park et al. 2014). During hypoxic and angiogenic stress, a substantial amount of different proteins get misfolded/unfolded and accumulated, which possibly trigger apoptosis of $f d-F L S$ in patients with RA (Park et al. 2014). In contrary, despite being modulated by increased ER stress, delayed apoptosis of $f d$-FLS is previously reported (Kabala et al. 2017). Autophagy, a highly conserved homeostatic mechanism, regulates the degradation of damaged cytosolic constituents (Chun and Kim 2018), which also induces cell death via apoptosis, thereby implicating the dual role of autophagosomal machinery (Kato et al. 2014). In comparison to patients with $O A$, higher ER stress mediated autophagy activation in RA demonstrated more apoptotic resistance in $f d$ FLS (Xu et al. 2015).

Methotrexate (MTX) - a DMARDS used as a treatment modality of patients with RA. Its efficacy varies from person to person, $50 \%$ of patients showed little response after administered this drug (Yu et al. 2018). Despite being toxic, a low dose of MTX is proven to be beneficial in inducing apoptosis in synovial cells (Herman et al. 2005). The effect of MTX upon the proliferation of monocytes and non-effectiveness in $f d$-FLS proliferation has been previously reported (Bergstrom et al. 2018). Theaflavin 3,3'di-gallate (TF3), an active compound of black tea, has the potential to prevent inflammation and induce apoptosis in numerous cell types both in malignancy and mouse model of RA (Liu and Li 2019) of inflammatory diseases including cancer (Tu et al. 2016). Our study aimed to evaluate the effect of TF3 (antiinflammatory and pro-apoptotic) along with low dose MTX and in combination (TF3 + MTX) upon cell proliferation/viability, markers of hypoxia, angiogenesis, the status of apoptosis, and associated ER stress mediated autophagy in $f d-F L S$ in patients with RA.

\section{Materials And Methods}

\section{Study design:}

The study population included patients with OA and RA with effusion in at least one knee joint. All RA patients fulfilled the ACR/EULAR classification criteria for RA (Aletaha et al. 2010). Patients were recruited from the outpatient department of Rheumatology and clinical immunology at the Institute of post graduate medical education and research (IPGME\&R), Kolkata, who have already been administrated with DMARDs with or without NSAIDs. Patients with any other rheumatological disease, DM, HTN, hypothyroidism, $\mathrm{BMI} \geq 25$, pregnancy, renal and/or hepatic impairment, receiving steroids or any antioxidants were excluded. This study was approved by the Institutional Ethics Committee (No: Inst/IEC/24.02.2014), and informed consent was obtained from all participants. Baseline demographic and clinical data were collected from all the patients. Approximately $10 \mathrm{ml}$ of Synovial fluid was aspirated from the knee joint of the participant maintaining strict aseptic measures.

\section{Cell culture and reagents:}


Isolated cells were cultured in DMEM (Dulbecco's Modified Eagle Medium, D5648Sigma, St Louis, MO, USA) supplemented with $10 \%$ fetal bovine serum/FBS (Invitrogen, Rockford, IL, USA), $1 \%$ penicillinstreptomycin $(10,000 \mathrm{U} / \mathrm{ml})$, [Cat no-15140122] from Invitrogen at $37{ }^{\circ} \mathrm{C}$ with $5 \% \mathrm{CO}_{2}$ in a humidified incubator. Theaflavin 3, 3'digallate/TF3 [Cat no-92223-1MG] and Methotrexate/MTX [Cat no-M8407] were purchased from (Sigma, St Louis, MO, USA). For protein estimation, Bradford reagents were obtained from bio rad (Cat no- 5000006). Primary antibodies for human [HIF-1a(ab51608),GRP78(\#3183), CHOP(\#2895), HSP-70(\#4876), Bcl-2(\#4223), Bax (\#2772), Beclin-1(\#3738) p62 (\#5114), a-tubulin (\#2144), 10x cell lysis buffer Cat no-\#9803 and Protease Inhibitor Cocktail, PKC (100x) Cat no-5871] were obtained from Cell-Signaling Technologies Inc. (Danvers, MA, USA). Nitrocellulose membrane was procured from MF-Millipore [Cat no- HAWP04700] $0.45 \mu \mathrm{m}$ pore size. Caspase-9 and Caspase- 3 were obtained from Biolegend (Cat No: 621902 and 634101 respectively). Anti-LC3b antibody (ab51520) was procured from Abcam. The concentration of VEGF-A and ANG-1 ELISA Kits [(ELH-VEGF-1) and (IQHAngiopoietin1-1)] were procured from (Ray Biotech, Norcross, GA). For detection of apoptosis FITC Annexin V Apoptosis Detection Kit, I (Cat no-556547) was used from BD, Biosciences.

\section{Culture of synovial fluid-derived fibroblasts:}

SF from patients with RA and OA was centrifuged (10X3000 rpm), the cell pellet was re-suspended in (\%) DMEM supplemented with $15 \%$ FBS, $2 \mathrm{mM} \mathrm{L-glutamine,} 50 \mathrm{U} / \mathrm{ml}$ penicillin, $50 \mu \mathrm{g} / \mathrm{ml}$ streptomycin, and plated in $25 \mathrm{~cm}^{2}$ flasks. After $24 \mathrm{~h}$, non-adherent cells were discarded. Spindle-shaped cells were observed after the third passage. The presence of $f d-F L S$ was confirmed by phenotypic characterization by flow cytometry using FITC conjugated CD90 (Stebulis et al. 2005).

\section{Extracellular cytokines measurement by ELISA:}

Extra-cellular pro-inflammatory cytokines (TNF-a, IL-6) along with angiogenic markers (VEGF-A and ANG1 ) as well as inflammatory marker CRP were measured from cell-free supernatant of cultured $f d-F L S$.

\section{Cell viability assay:}

The pro-apoptotic activity of TF3 alone and in combination with MTX was evaluated in $f d$-FLSobtained from RA patients by MTT assay (Sen et al. 2007). Briefly, $5 \times 10^{4}$ cells $/ \mathrm{ml}$ were seeded in 96 well plates in DMEM medium (supplemented with 10\% FBS and antibiotics) and incubated with TF3 (0-50 $\mu \mathrm{M})$ respectively for $48 \mathrm{~h}$.For combinatorial treatment, one dose of MTX (125nM) was fixed with the variable dose of $\operatorname{TF} 3(5,10,15,20,30 \mu \mathrm{M})$ for $24 \mathrm{~h}$. After treatment incubation, $f d$-FLS viability was measured using 3-(4, 5 dimethylthiazole-2-yl)-2, 5-diphenyltetrazolium bromide, and the optical density (OD) was measured at 570nM in a microplate reader (Byonoy absorbance 96 plate reader). The results were expressed at $\mathrm{IC}_{50}$ values i.e. the concentration that inhibited $50 \%$ of cell growth, enumerated by graphic extrapolation using GraphPad PRISM software (version 5).

\section{Identification of CD90+ cell by flow cytometry and analysis of apoptosis:}


Isolated cells were treated for $24 \mathrm{~h}$ with both MTX and TF3, followed by washing twice with 1xPBS. Cells $\left(1 \times 10^{6} \mathrm{cells} / \mathrm{ml}\right)$ were seeded in $1 \times$ PBS. Cells were then centrifuged for $10 \mathrm{~min}, 3000 \mathrm{rpm}$, the cell pellet was resuspendedin $100 \mu \mathrm{l}$ of (1x) annexin $\mathrm{V}$ binding buffer and kept for $10 \mathrm{~min}$, dark, RT. FITC conjugated annexin $\mathrm{V}$ and propidium iodide (PI) was added, kept for $15 \mathrm{~min}$ and 5 min respectively, dark, RT(Room temperature). Cells were acquired and analyzed in FACS Verse ${ }^{\mathrm{TM}}$ (BD Bioscience) after the addition of $400 \mu$ l of binding buffer. Each experiment was performed in triplicate.

\section{RNA isolation and cDNA synthesis:}

Total RNA was extracted from synovial fluid-derived synovial fibroblast cells using $300 \mu \mathrm{I}$ TRIzol ${ }^{\mathrm{TM}}$ (Invitrogen, Carlsbad, CA, USA) at a ratio of (1:1). Cells were slightly flush and stored overnight at $-20^{\circ} \mathrm{C}$. On the next day, the mixture was centrifuged at $10,000 \mathrm{rpm}$ for $5 \mathrm{~min}$ at $4^{\circ} \mathrm{C}$. The clear supernatants were transferred to a fresh microcentrifuge tube and allowed to stand for $5 \mathrm{~min}$ at room temperature. Next, $200 \mu \mathrm{l}$ of chloroform was added and shaken vigorously. Then the mixture was allowed to stand at room temperature for 2-5 min and was again centrifuged at 10,000 rpm for $10 \mathrm{~min}$. The exact upper aqueous phase was then transferred to a fresh tube followed by the addition of $0.5 \mathrm{ml}$ of isopropanol and was again subjected to vigorous shaking. The mixture was then kept at room temperature for 5-10 min. Centrifugation was carried out at $10,000 \mathrm{rpm}$ at $4^{\circ} \mathrm{C}$ to settle down the pellet from the mixture. The RNA pellet was collected, washed with $75 \%$ ethanol followed by centrifugation at 10,000 rpm, and allowed to air dry at room temperature. Finally, the dried RNA pellet was re-suspended in DEPC water and quantified in a nano-drop spectrophotometer (Thermo Scientific, USA). Approximately, $1 \mu \mathrm{g}$ of cDNA was prepared from isolated RNA with a total volume of $11.5 \mu$ (RNA+DEPC water). Next, oligo dT (1 $\mu$ l) was added and incubated at $70^{\circ} \mathrm{C}$ for 5 min inside the PCR machine (Applied Biosystems, USA). The mixture was taken out and placed in chilled-ice for $5 \mathrm{~min}$ followed by the addition of PCR master mix. The mixture was given a short vortex to mix well followed by incubation at $37^{\circ} \mathrm{C}$ for $5 \mathrm{~min}$. Then, $1 \mu \mathrm{l}$ of reverse transcriptase was added and incubated at $42^{\circ} \mathrm{C}$ for 60 min inside the PCR machine. Finally, the mixture was again incubated at $70^{\circ} \mathrm{C}$ for $10 \mathrm{~min}$ after that the mixture was taken out and kept on ice for 5-10 min and stored at $-20^{\circ} \mathrm{C}$ for further processing.

\section{Reverse transcriptase (RT)-PCR:}

The gene expressions of the prepared cDNA samples were analyzed by real-time quantitative PCR analyses. The reactions were carried in $0.2 \mathrm{ml}$ strip tubes/96well plates (Applied Biosystems, USA) having a final reaction volume of $10 \mu \mathrm{l}$. The reaction mixture containing $10 \mathrm{ng}$ cDNA, $2 \mathrm{ng}$ of both forward and reverse primers along with 1X SYBR-Green PCR master mixture (Power SYBR-Green PCR Master Mix; Applied Biosystems, USA) was prepared. The quantitative real-time PCR was carried out using RTPCR system (Applied Biosystems, USA). All the reactions were performed in triplicates and the expression of enzyme GAPDH was used as a housekeeping gene for the following experimental studies. Primer sequences for spliced XBP-1 mRNA was forward 5'-CCCTCCAGAACATCTCCCCAT-3' and reverse 5'ACATGACTGGGTCCAAGTTGT-3' and unspliced sequences was forward 5'-CAGACTACGTGCACCTCTGC-3' and reverse 5'-AGTTGTCCAGAATGCCCAACA-3'. Primer sequences for IRE1-a were forward 5'- 
CCGAACGTGATCCGCTACTTCT-3' and reverse 5'-CGCAAAGTCCTTCTGCTCCACA-3' (IDT Inc., Singapore).

The reaction mixtures were incubated at $95^{\circ} \mathrm{C}$ for $2 \mathrm{~min}$, followed by 40 cycles of $5 \mathrm{sec}$ at $95^{\circ} \mathrm{C}$ and 30 $\mathrm{sec}$ at $60^{\circ} \mathrm{C}$ respectively (Ahmadiany et al. 2019). Relative expression of transcript levels of each sample was demonstrated according to the $2 \Delta \Delta \mathrm{CT}$ methods.

\section{Immunoblotting:}

After washing twice with 1X PBS, cultured $f d$-FLS were lysed in cell lysis buffer (1X) contains PKC (Protein Kinase C). Quantification of total protein was done using Bradford reagent. Cell lysate proteins $(50 \mu \mathrm{g})$ were separated by SDS-polyacrylamide gel electrophoresis, transferred into nitrocellulose membrane, and (3\% BSA dissolved in Tris buffer saline or TBS) was used for blocking for $1 \mathrm{hr}$, RT and incubated overnight at $4^{\circ} \mathrm{C}$ with desired $1^{0}$ antibodies (Dilution- 1:10000). After washing twice with TBST (Tris Buffer solution with Tween), the membrane was incubated with HRP conjugated $2^{0}$ antibody (Dilution1:500) for $2 \mathrm{hrs}$, RT. The membrane was washed four times with TBST followed by development via chemiluminescence using a gel documentation system (Bio-Rad-Chemidoc, XRS+), quantified by Image J, (Image J, 1.40, USA), and normalized by a-tubulin for further analysis.

\section{Caspase 3 assay:}

Caspase-3 activity in synovial fibroblast cells was detected by using a caspase-3 assay kit [(BioVision, Sandiego, USA) (cat no: K106)]. Cells were seeded in a 6 well plate at $1 \times 10^{4}$ cells/well, incubated overnight with a combination of MTX $(125 \mathrm{nM})$ and TF3 $(10 \mu \mathrm{M})$ for $24 \mathrm{~h}$. After completing the treatment, cells were lysed using cell lysis buffer (cell signaling) and $2 X$ reaction buffer was added to each sample with $5 \mu$ l of DVD-pNA substrate, and cells were incubated at $370 \mathrm{C}$ for $1-2 \mathrm{~h}$, followed by measuring by spectrophotometer at 400-405nM.

\section{Immunofluorescence staining and confocal microscopy:}

Synovial fibroblast cells were seeded $\left(1 \times 10^{6} \mathrm{cells} / \mathrm{ml}\right)$ in a confocal dish (SPL Life Sciences) and incubated overnight. After treatment with [either rapamycin or MTX+TF3 and rapamycin (200nM)], cells were fixed with $4 \% \mathrm{v} / \mathrm{v}$ paraformaldehyde (in 1x PBS) at RT, washed twice, permeabilized with $0.2 \%$ triton X-100 in 1x PBS for 20 mins at RT. Cells were then blocked with 2\% BSA, washed, and incubated overnight with primary antibody LC3B (ab51520) (1:100 dilution) at $4^{\circ} \mathrm{C}$. Cells were treated with fluorophore conjugated secondary antibody (Invitrogen) and Hoechst (for nuclear staining, Invitrogen) diluted in 1\% BSA - 1x PBS and viewed under Olympus FV10i-LIV confocal laser-scanning microscope (magnification: $60 * 4 X / 1.35$ ).

\section{Statistical analysis:}

Data are expressed as mean \pm SD or median (inter quartile range, IQR). Student t-test has been done for parametric data, for non-parametric data paired t-test (Mann Whitney U test) were done. Repeated measures ANOVAs was done for more than two data set. The correlation was done using Pearson's 
correlation coefficient test for parametric data and Spearman rank correlation coefficient for nonparametric data using Graph Pad Prism software, version 5.0, (Graph Pad Software Inc, La Jolla, CA, USA). $p<0.05$ was considered as significant for all statistical analysis.

\section{Results}

\section{Clinical characterization of RA and OA patients:}

Patients with active RA $(n=11)$ and OA $(n=10)$ were enrolled in this study. Comparative analyses of clinical features demonstrating inflammatory markers like CRP (C-reactive protein) and Anti-CCP were significantly higher in RA compared to OA ( $p<0.001$ and $p<0.001$ respectively) in SF from knee joints suggesting inflammation was predominant in patients with RA [ Table 1].

\section{fd-FLSexhibits an overexpressed levels of inflammatory markers:}

Immunophenotyping characterization of $f d-F L S$ was conducted by flow cytometric assay. Isolated $f d-F L S$ (from SF) sourced from knee joints of RA and OA patients were stained with anti-human CD90 FITC. The result showed that the frequency of $C D 90^{+}$population was higher in $f d-F L S$ of $\mathrm{RA}(p<0.01)$ than in $\mathrm{OA}$ [91.90 (88.16-95.73) \%, vs 4.005 (2.423-8.925) \% (Fig. 1A i, ii and B)]. Inflammatory marker like CRP was significantly upregulated ( 31 folds) in $f d-F L S(p<0.01)$ of patients with RA compared to OA [57.80 (16.95-55.73) vs. $2.9(1.42-8.92) \%$, Fig. 1C]. No significant correlation was found between CRP and $\mathrm{CD} 90^{+}$population in patients with $\mathrm{OA}[\mathrm{Fig} .1 \mathrm{D}$, i] whereas the frequency significantly varied in patients with $\mathrm{RA},[\mathrm{r}=0.7, p=0.007$, Fig. 1D, ii $]$.

Level of pro-inflammatory markers like TNF-a was significantly upregulated in RA compared to OA ( $p<$ $0.001)[856.0(528.0-952.3)$ vs. $6.50(3.88-16.35) \%]$, and IL-6 showed a similar expression pattern [548.06(154.01-577.0) vs. 5.90(3.57-7.00) \%] in cell-free supernatant of $f d-F L S$ [Fig. 1E i, ii]. Frequency of CD90 ${ }^{+}$population in $f d-F L S$ positively correlated with TNF-a $(r=0.6, p=0.03)$ and IL-6 $(r=0.6, p=0.02)$ in patients with RA [Fig. 1E iv, vi] while OA patients did not correlate [Fig. 1E iii, v].

\section{Combination treatment of TF3 with MTX reduces the cell viability offd-FLS sourced from SF of patients with RA:}

The anti-proliferative effects of TF3 and/or MTX or in a combination of both on $f d$-FLS were determined by MTT assay. RA-FLS were treated with TF3 $[0-50 \mu \mathrm{M},(5,10,15,20,30,40,50 \mu \mathrm{M})]$ for 18,24 and $48 \mathrm{~h}$. Cell viability was determined at $\mathrm{IC}_{50}$ dose at respective time points. We found that TF3 administration at $20 \mu \mathrm{M}$ dose for $24 \mathrm{~h}$ was most effective in reducing the cell viability compared to other time points [Fig. 2A]. Further increasing the TF3 dose above 20 $\mu \mathrm{M}$ (4-6 days) we furnished no change in cell death and saturation was reached at $48 \mathrm{~h}$ time point. MTX $(1 \mu \mathrm{M})$ was able to diminish $50 \%$ of cultured $f d-F L S$ [Fig. 2B] when incubated for $24 \mathrm{hr}$. Next, we fixed the $\mathrm{IC}_{25}$ dose of MTX and determined the optimum effective dose of TF3 in combination on $f d-F L S$ isolated from RA for 24hr. Our investigation showed 
effective minimal IC $\mathrm{C}_{50}$ concentration of TF3 was $10 \mu \mathrm{M}$ over a fixed dose of MTX $\left(125 \mathrm{nM}, \mathrm{IC}_{25}\right)$ [Fig. 2C]. Based on these findings, we chose TF3 $(10 \mu \mathrm{M})$ in combination with MTX $(125 \mathrm{nM})$ for our further studies.

\section{Combination of MTX and TF3 downregulates the HIF-1a expression infd-FLSfrom SF of RA patients:}

Inflammation associated with uncontrolled cell proliferation is one of the causes for hypoxic stress generated in the cellular microenvironment (Qu et al. 2018), which is a regular phenomenon in RA. We investigated the expression of hypoxic stress in RASF derived $f d-F L S$ after treatment with MTX and TF3. It was observed from immunoblot analysis that $f d-F L S$ (without treatment) had a profound expression of HIF-1a enzyme suggesting that HIF-1a might be induced under chronic pro-inflammatory stress [Fig. 3A]. Treatment with MTX in combination with TF3 $(10 \mu \mathrm{M}, 24 \mathrm{~h})$ reduced the expression of HIF-1a significantly $(p<0.001)$ compared to their individual administration $(p<0.05$ for MTX and TF3) in $f d$-FLS obtained from SF of RA [Fig. 3A-B].

Extensive expression of HIF-1a associated with inflammatory diseases triggers the downstream signaling molecules like VEGF and Ang-1 (Krock et al. 2011). As expected, we observed an increased level of VEGF in $f d$-FLS from RA patients compared to OA $(p<0.05)$ where combinatorial treatment (MTX and TF3) showed a significant decline in VEGF level [ $p<0.001$, Fig. 3C i]. Similarly, the Ang- 1 level was significantly $(p<0.05)$ higher in RA compared to OA, which was drastically declined upon dual administration of MTX and TF3 in $f d$-FLS $[p<0.01$, Fig. $3 C$ ii]. Collectively these data suggested that MTX and TF3 in combination might reduce the angiogenic potency of $f d-F L S$ in RA patients.

\section{'Add on' effect of TF3 with MTX upon hypoxia induced ER stress infd-FLSfrom RA patients:}

Earlier reports suggest that inflammation mediated hypoxic stress within fibroblasts is often associated with the development of unfolded protein response (UPR) and ER stress (Park et al. 2014). To address this, we analyzed the expression of well-established ER stress marker, GRP78 in $f d F L S$ of different studied groups. Our immunoblot data demonstrated an overexpression of GRP78, in $f d$-FLS sourced from patients with RA compared to OA [Fig. 4A] as represented in density scan data [ $p<0.05$ ] [Fig. 4B i]. Individual administration of MTX and TF3 was noticeably less effective in reducing the expression of GRP78 and consequently, hypoxia-mediated ER stress compared to combinatorial administration which efficiently hindered ( $\nabla 5$ fold) the expression of GRP78 in $f d F L S$ of RA than OA. Likewise, heat shock protein 70 (HSP70) (a molecular chaperone), which is also known to be an exquisite intracellular marker of ER stress associated RA pathogenesis (Luo et al. 2008) was also assessed in our study. Data showed the expression of HSP70 followed a similar trend as observed in GRP78 in $f d$-FLS ( 3.43 fold) of RA compared to OA, $p<0.05$, [Fig. 4B ii]. MTX + TF3 efficiently reduced the HSP70 expression in $f d-F L S$ from RA than individual application (Luo et al. 2008).

Under UPR-stress, the downstream transduction of signaling milieu from GRP78 is emanated primarily in three directions (Wang et al. 2009). One of which is inositol-requiring transmembrane kinaseendoribonuclease-1 a IRE1 a, receives the pro-apoptotic signal. ER stress causes oligomerization and activation of the kinase domain in IRE1a (Junjappa et al. 2018). Activation of kinase domain associated 
RNase domain in IRE1 a splices X-binding protein 1 (XBP1) mRNA. This spliced mRNA in turn regulates ER associated genes especially apoptosis (Wu et al. 2015). In our study, we evaluated the expression of IRE1a and spliced XBP-1 in $f d-F L S$ of RA patients. The result showed that expression of IRE1a was significantly upregulated in $f d-F L S$ of RA whereas combined treatment with TF3 and MTX markedly reduced the expression much closer to control cells (OA) [Fig. 4C i]. Similarly, the mRNA level of spliced XBP-1, was quite over expressed in $f d-F L S$ from RA patients than OA, and after administration of MTX + TF3 [Fig. 4C ii] the expression level of spliced XBP-1 was drastically declined close to fd-FLS from OA than their individual treatments. It is already known that spliced XBP-1 mRNA acts as a transcription regulator and modulate expressions of various pro and anti-apoptotic genes. One of the prominent genes is pro-apoptotic protein CCAAT enhancer-binding protein homologous protein (CHOP) which is activated by spliced XBP-1, initiates the apoptotic process (Yang et al. 2017). Immunoblot analysis of CHOP showed that it was down-regulated in $f d$-FLS derived from RA compared to OA patients. Combinatorial administration of MTX and TF3 significantly $(p<0.001)$ enhanced the CHOP expression $(5$ fold $)$ compared to their individual application $(p<0.001)$, [Fig. 4D i-ii]. Conclusively these findings suggest that MTX and TF3 co-administration might induce the ER-stressed $f d F L S$ to receive a pro-apoptotic stimulus.

\section{Combination therapy of TF3 with MTX induces apoptosis infd-FLS derived from RA patients by inducing BAX and suppressing Bcl-2:}

fdFLS from RA patients exhibit resistance to ER stress mediated cell death (Park et al. 2014; Ahmadiany et al. 2019). Our findings showed that in RA fd-FLS, CHOP was down-regulated which might, in turn, modulate the status of apoptosis. Hence, to investigate this, we assessed the statuses of anti-apoptotic protein (Bcl-2) and pro-apoptotic Bax (Bcl-2 associated X protein) since they are being targeted by $\mathrm{CHOP}$ directly (Hu et al. 2018). Immunoblot data showed that Bcl-2 expression was concomitantly decreased after application of individual drug therapy to $f d$-FLS from RA [Fig. 5A, B i]. The most significant decrease in expression was observed after combination treatment with MTX and TF3. On the other hand, the expression of Bax was found to be low in untreated $f d$-FLS cells but administration of combination therapy with MTX and TF3 reversed the expression significantly thereby indicating initiation of the proapoptotic signals [Fig. 5A, B ii].

Next, we examined the impact of Bax on programmed cell death and its consequences after administration of combination therapy with MTX and TF3 in fd-FLS from RA patients. Findings showed that the frequency of Annexin $\mathrm{V}^{+}$apoptotic cells was 5.450 (4.030-5.640) in untreated $f d$-FLS from RA patients. Administration of MTX $(125 \mathrm{nM})$ and TF3 $(5 \mu \mathrm{M})$ in combination increased the frequency of apoptotic cells [15.37 (14.56-16.50)] compared to their individual application which was further extrapolated from the percentage of apoptosis (Fig. 5C i-ii). MTX + TF3 in combination treatment on caspases (initiator and executioner caspases) was also determined. Immunoblot study showed that the expressions of both initiators (caspase 9) as well as executioner (caspase 3) (total and cleaved) were down-regulated in $f d$-FLS of RA compared to OA (Fig. 5D ii, i). Both cleaved caspase 3 and caspase 9 expressions were increased significantly (5 fold and 6 fold respectively) after dual administration of MTX with TF3 $(p<0.05)$ in $f d$-FLS from RA than untreated $f d$-FLS (Fig. 5iii-iv). The activity of caspase 3 was 
also monitored by colorimetric assay. MTX + TF3 treatment restored the activity of executioner caspase in $f d-F L S$ from RA than untreated cells, $(p<0.01)$ (Fig. 5E).

\section{MTX and TF3 administration synergistically inhibit ER stress induced autophagy infd-FLSderived from RA patients:}

In RA $f d$-FLS, under ER stress, a halt in the apoptotic pathway is often linked with activation of autophagosomal proteins leading to prolonged cell survival (Vomero et al. 2018). Dissociation of antiapoptotic Bcl-2 from Beclin-1 (known to be a prime regulatory molecule in the autophagy startup process) induces autophagy (Decuypere et al. 2012) (Regulation of the Autophagic Bcl-2/Beclin 1 Interaction). To evaluate the expression of Beclin-1 in the modulation of the apoptotic pathway we treated the cells with MTX and TF3 and quantified them by immunoblot assay. The image showed that Beclin-1 in untreated $f d$ FLS was highly expressed than cells from OA patients [Fig. 6A \& B]. Individual treatment with MTX/TF3 was not strong enough in suppressing the expression of Beclin- 1 in $f d$-FLS compared to their combined application which was quite evident from their densitometric scans. The densitometric ratio between Beclin1 and Bcl-2 was measured to assess the switching over between apoptotic and autophagic pathway. Data suggested a significant decrease in beclin: Bcl-2 ratio in $f d$-FLS from RA indicating a shift to the autophagic pathway. MTX-TF3 treatment to RA-fd-FLS reversed the shifting towards programmed cell death pathway significantly compared to the individual drug administration.

We also examined the expressions of autophagic inducer proteins like LC3B and p62 in $f d$-FLS obtained from RA and OA. Data showed that both LC3B and p62 were highly expressed in untreated $f d$-FLS from RA compared to OA (Fig. 6A,C,D). Treatment with MTX or TF3 alone was less effective in reducing the expression of both of these proteins than combination therapy as also prominent from relative band intensities data (Fig. 6A,B,C\&D). These findings suggested that TF3 in combination with MTX might play a crucial role in downregulating the expression of Beclin-1, p62, LC3B thereby might halt the entry to autophagy. Further analysis was done to evaluate the mode of action of LC3B protein. It is known that migration of this protein to the nucleus is necessary to trigger autophagic activity (Huang and Liu 2015). Confocal micrographs showed accumulation of LC3B within the nucleus (red dots) of $f d-F L S$ from RA patients (Fig. 6E). Individual MTX and TF3 treatment reduced the accumulation of LC3B which was not significant (Data not Shown). Rapamycin which is one of the inducers of autophagy was administered which significantly increased autophagic puncta (Andersson et al. 2016) whereas combination treatment with rapamycin depicted lesser accumulation of LC3BI protein within the nucleus of $f d$-FLS from RA suggesting a reduction in autophagic activity.

\section{MTX + TF3 treatment halt the autophagy and induce apoptosis infd-FLSfrom RA:}

This finding instigated us to see whether inhibition in autophagic proteins diverts the cellular physiology to apoptotic death or not. To investigate this, we treated the $f d-F L S$ from both RA and OA with autophagic blocker 3-MA and estimated the apoptotic events before and after the treatment with individual and/or combination therapy. Results demonstrated that $f d-F L S$ treated with 3-MA increased cell death frequency being [14.48(12.07-19.18)] compared to baseline value [6.70 (4.000-9.700)] (Fig. 7A). On the other hand, 
individual treatment of MTX or TF3 didn't show any effective result, frequency being [15.76(12.05-18.73) vs. 18.91(16.43-20.74)]. A drastic increase in apoptotic frequency was demonstrated when 3-MA administration with TF3 or MTX in fd-FLS from RA, frequency being [31.01(24.50-38.31] (Fig. 7A-B).

\section{Discussion}

Cell proliferation and resistance towards apoptosis are one of the hallmark incidences that takes place in RA synovium (Nygaard and Firestein 2020). Autophagy is known to be a protective mechanism against the pro-apoptotic signal (Heymann 2006) but many have shown its association in inducing programmed cell death especially in cancer cells and other inflammatory diseases, etc. (Jung et al. 2020). In RA, the fdFLS are known to evade apoptotic signals by inducing autophagy (Xu et al. 2015) thereby continue their survival and pannus expansion (Zhu et al. 2017). Prolonged accumulation of inflammatory loads due to infiltration of pro-inflammatory cytokines and concomitant building up of hypoxic stress promote two vital incidents. One of which is the release of angiogenic factors due to hypoxia and the onset of intracellular stress accumulation. ER stress is one such incident that takes place in the RA synovium where due to hypoxic and inflammatory loads, cells are forced to destabilize their protein folding machinery.

Among the conventional medications, MTX has always remained a choice of first-line defense against RA (Pincus et al. 2003). Recent studies intimidated that prolonged administration of conventional treatments like MTX, DMARDs (Methotrexate, leflunomide, hydroxychloroquine, and sulfasalazine) initiate side effects like liver toxicity etc. (Wang et al. 2018). In RA, the anti-inflammatory effect of MTX is chiefly exerted by Adenosine release. Originally, MTX acts as a folate inhibitor, blocks the dihydrofolate reductase activity, usually recommended for treatment of leukemia at a very high dose (Sakura et al. 2018). Inhibition of purine metabolism by halting the cell cycle movement at the $S$ phase causing apoptosis is another predominant pathway through which it acts (Goncharova and Frankfurt 1976; Fairbanks et al. 1999). However, the drugs show its efficacy at a very low dose in RA (Steinsson et al. 1982). In RA, it is highly elusive to ascertain that whether a low dose of MTX arrests the cell cycle and initiates caspases activities or not. On the other hand, a higher dose of MTX is not recommended to administer in RA patients due to its severe complications (Bello et al. 2017). On keeping these conditions, we chose our primary drug to be MTX (low dose) and decided to co-administer a compound, which will be low in its side effects, but be effective in inducing pro-caspases activities thereby intensify the MTX effect.

Polyphenolic derivatives from black tea have therapeutic properties against several human disorders ranging from metabolic to cardiovascular and cancer (Khan and Mukhtar 2013). The mode of actions of these therapeutics generally ranges from anti-oxidative, anti-inflammatory to pro-apoptotic effects (Gosslau et al. 2018). TF3, an active compound of black tea exhibits the strongest anti-inflammatory, anti-angiogenic and pro-apoptotic properties in different cell types of various disease models (Gao et al. 2016). Thus, the study was conducted to investigate the efficacy of polyphenolic derivative of black tea (TF3) in combination with MTX (low dose) in modulating the angiogenesis and autophagy-mediated cell 
proliferation in $f d$-FLS from RA patients. It is well evident from other studies and as well as from ours that levels of pro-inflammatory cytokines (TNF-a, IL-6) are highly expressed in synovial fluid of RA patients as OA. Our findings also revealed that increased expressions of pro-inflammatory markers and CRP levels have a positive association with an increased $f d$-FLS expression in RA synovial fluid than OA. A substantial increase in $f d-F L S$ population within synovial joints might reflect persistence of inflammation as also identified by other studies (Alunno et al. 2017). Our finding also showed that administration of a low dose of MTX in combination with TF3 was more efficient in killing the cells ( $f d-F L S$ ) and downregulated the inflammatory loads compared to individual administration (TF3/MTX). This observation is well consistent with the anti-proliferative, anti-inflammatory and pro-apoptotic action of TF3 on inflammatory and tumor cells (Tu et al. 2016). The proliferation of synovial fibroblasts in RA is often associated with building up of hypoxic stress (Quinonez-Flores et al. 2016), which causes the release of angiogenic factors within synovium (Elshabrawy et al. 2015). HIF-1a and several other cytokines like TNF-a, IL-1 $\beta$ instigate $f d$-FLS to release VEGF and Ang-1 in synovial joints (MacDonald et al. 2018) which was evident from our observations in RA than OA. Therefore, the RA fd-FLS has greater scope to be stimulated by these molecules than in OA. TF3 has potent anti-angiogenic action on several cell types, especially on tumor cells where it hinders the expressions of hypoxic and angiogenic factors (Gao et al. 2016). Notably, we also found a significant downregulation of HIF-1a, VEGF, and Ang-1 factors in $f d$-FLS from RA after TF3 + MTX treatment.

Concomitant accumulation of pro-inflammatory burdens and hypoxic stress in $f d$-FLS trigger downstream UPR and subsequent ER stress which play a pivotal role in disease pathogenesis and progression (Park et al. 2014). In rheumatic diseases, the challenging hypoxic microenvironment within synovium, proliferative $f d$-FLSs are induced to accumulate misfolded proteins, which in turn cause UPR and upregulation of ER stress factors like GRP78, HSP70, and CHOP (Yoo et al. 2012). GRP78 is a molecular chaperon that exhibits anti-apoptotic properties against numerous inflammatory, tumourigenic and rheumatic diseases and serves as a pivotal factor in RA pathogenesis (Yoo et al. 2012). We observed a significant elevation of the GRP78 level in the RA $f d$, but not in OA. It is implicated that GRP78 in $f d$-FLS could serve as a therapeutic target (Park et al. 2014). Likewise, another essential molecular chaperon HSP70 is already been known to be elevated in RA fd-FLS (Kang et al. 2009) which was well consistent from our immunoblot study. The level of HSP70 was reduced after TF3 alone or TF3 + MTX combination. These two findings indicate the efficacy of combination therapy is declining the expressions of ER sensor proteins and ER stress response.

During ER stress, the activated GRP78 conveys signal downstream to a crucial molecule, IRE1a that holds the key for enzymatic activity leading to cell survival or death (Ahmadiany et al. 2019) via another regulatory molecule XBP-1 mRNA. The spliced XBP-1 mRNA is responsible for inducing apoptotic signals (Zeng et al. 2009). Our results furnished that expressions of IRE1a and XBP-1 were elevated in $f d-F L S$ of RA patients as observed by others also (Kabala et al. 2017). However, there are evidences suggesting, elevated XBP-1 level contributes to an apoptosis resistant phenotype in RA fd-FLS (Ahmadiany et al. 2019). In our work, the levels of IRE1a and sXBP-1 were compromised after MTX + TF3 application thereby highlighting a probable pro-apoptotic activity of TF3. The pro-apoptotic induction ability of TF3 + 
MTX combination was further verified by monitoring another downstream sensor protein CHOP which is responsible for caspases activation. Previously, it has been shown that RA $f d$-FLS exhibits a lower expression of CHOP compared to OA $f d$-FLS, as observed in ours that further justifies apoptosis-resistant characteristic in RA $f d$-FLS (Bustamante et al. 2017).

Since $\mathrm{CHOP}$ is a transcription factor, it is known to regulate numerous anti-apoptotic factors especially $\mathrm{Bcl}-2$ and pro-apoptotic like Bax (Hu et al. 2018). We furnished an overexpressed BCL-2 and underexpressed Bax in $f d-$ FLS from RA implicating the importance of $\mathrm{CHOP}$ in this regard. On the other hand, TF3 or TF3 + MTX reverted the expressions of $\mathrm{Bax}$ and $\mathrm{Bcl}-2$ through upregulating $\mathrm{CHOP}$ which can be predicted as a switching from anti to pro-apoptotic phenotype in RA fd-FLS. This chemo-therapeutic activity of TF3 by maneuvering Bcl-2/Bax has previously been argued in cancer and inflammatory cells (Leone et al. 2003). Modulation of caspases (both initiator and executioner) activities in RA $f d$-FLS is a well-known mechanism by which apoptosis is halted and localized cellular proliferation continues (Smith and Walker 2004). TF3 + MTX induced the caspases activities and apoptosis in $f d$-FLS as observed in our findings. Previously, TF3 has been shown to promote apoptotic genes in combination with phytochemicals or other drugs in various cancer and inflammatory cells (Pan et al. 2018) which is consistent with our findings.

Inhibition in the natural apoptotic pathway in RA $f d$-FLS instigated us to speculate the role of autophagy in their survival. Since, it is recently been known that in RA $f d-F L S$ autophagy acts as a protective mechanism against apoptosis where knocking down the autophagic genes promotes apoptosis (Vomero et al. 2018). Beclin-1 is one such factor whose modulation in RA $f d$-FLS causes persistence of autophagy mediated survival (Xu et al. 2015) as observed from our immunoblot analysis. Another important factor, LC3B works downstream of Beclin-1 regulates autophagic process where LC3B-I converts to LC3B-II after accumulation in autophagosome (Vomero et al. 2018). LC3B-II thereafter becomes the structural entity of autophagosomal vesicle. We observed elevated levels of LC3B-II/: LC3B-I in RA $f d$-FLS, indicating an augmented autophagy pathway in RA. These observations were similar to the findings of (Zhu et al. 2017). p62 is an adapter protein whose binding is necessary for the transformation of LC3B-I to LC3B-II forming autophagmosomal bodies (Runwal et al. 2019). Higher expression of p62 in our data indicated autophagmosomal vesicles were formed in RA $f d$-FLS. Individual MTX or TF3 or combined treatment showed their ability to reduce autophagic proteins like LC3B-I, LC3B-II, and p62. Many researchers observed that isolated TF3 administration has the ability to induce autophagic flux by modulating LC3B-II and Beclin-1 levels in different cell types and cause cell death which contradicted our findings (Shen et al. 2019). Although these findings only suggest that autophagy has role in promoting cell death, which is inconsistent with $f d$-FLS in RA, where apoptosis is restricted by autophagy. TF3 + MTX in this regard, might justify its role in inducing apoptosis by halting autophagy. This phenomenon was further justified by applying autophagic inducer (rapamycin) to $f d$-FLS along with TF3 and MTX to show their involvement in restricting autophagy. These molecules proved to be successful in reducing the level of autophagosomal proteins LC3B-II accumulation as confirmed by confocal imaging. This may be predicted as the modulation of the functionality of Beclin-1 and p62 under combination therapy. Induction of apoptosis by blocking autophagy in $f d$-FLS from RA using our treatment was confirmed 
using autophagy blocker 3MA. MTX and TF3 combination showed their ability to promote apoptosis in 3 MA mediated autophagy restricted $f d$-FLS from RA, which justifies their apoptosis induction ability by restricting autophagy-mediated cell survivability in $f d$-FLS.

\section{Conclusion}

Overexpression of the pro-inflammatory cytokines and hypoxic stress induces the pathological alterations in $f d$-FLS from RA, leading to angiogenesis, cell proliferation, and dysregulated cell survival by modulating autophagy and apoptosis regulatory proteins. Co-administration of TF3 with MTX downregulated the angiogenic and hypoxic markers (HIF1- $a$, VEGF, and Ang-1) in RA $f d$-FLS, consequently counteracting the hypoxia-mediated ER-stress response and its imbalanced downstream pathway. Upregulation of ER stress-induced autophagy and downregulation of apoptosis in RA $f d$-FLS was significantly halted by the MTX and TF3 combination treatment, as evident from the pre and posttreatment assessment of the respective markers and, finally supported by 3-MA administration with TF3 or MTX in $f d-F L S$. From the pathologic viewpoint, disruption of the abnormal autophagy and apoptotic pathway in RA $f d$-FLS by MTX and TF3 combination is an important finding, as it may provide additional benefit to MTX monotherapy in limiting the synovial inflammation in RA. Further studies in this aspect are required to derive a definite conclusion.

\section{Abbreviations}

ANG-1: Angiopoietin 1,a- tubulin: Alpha tubulin, Caspase: Cysteine-aspartic proteases ,CRP: C-reactive protein, CHOP: C/EBP homologous protein, DMARDs: Disease-modifying antirheumatic drugs, ELISA: Enzyme-linked immunosorbent assay, ER-Stress : Endoplasmic reticulum stress, FBS: Fetal Bovine serum ,FITC : Fluorescein isothiocyanate,FLS: Fibroblast like synoviocytes, GAPDH- glyceraldehydes-3 phosphate dehydrogenase, GRP-78: 78-kDa glucose-regulated protein, HIF-1 a: Hypoxia-inducible factor 1alpha, Hsp70: Heat shock protein 70, IL-6: Interleukin 6, LC3b: Microtubule-associated proteins 1A/1B light chain 3B, MTT: 3-(4,5-dimethylthiazol-2-yl)-2,5-diphenyl tetrazolium bromide, MTX: Methotrexate, NSAIDs: Non-steroidal Anti-inflammatory Drugs, p-62: Sequestosome 1(SQSTM1), RA: Rheumatoid Arthritis, RT-PCR- Real time polymerase chain reaction, RT- Room temperature, SF-Synovial fluid, TF3: Theaflavin - 3,3' di gallate, TNF-a: Tumor necrosis factor-alpha, VEGF: Vascular endothelial growth factor,3-MA: 3-Methyladenine, IRE1 A-Serine/threonine-protein kinase/endoribonuclease IRE1a, XBP-1-X box-binding protein 1 .

\section{Declarations}

Funding sources:- This work was carried out with the support of funding agency National Tea Research Foundation (NTRF 174/2015).

Conflict of Interest:- The authors declare that there is no conflict of interests. 
Availability of data and material:- Not applicable

Code availability:- Not applicable

Author contributions:- S. Misra, A. Sarkar, S. Mondal, P.SM., A. Ghosh, P.C. and M.C. initiated the concept and designed the experiments. S. Misra, A.B, D.B., Sudipta C., Sulagna C., A. Saha performed the experiments. S Niyogi assisted in confocal experiments. S Misra, A Sarkar, S Mondal, A.B, P.SM. and A. Ghosh wrote the manuscript. A.C. extracted the synovial fluid from patients and assisted in manuscript preparation.

Ethics approval:- This study was approved by the Institutional Ethics Committee (No: Inst/IEC/24.02.2014) dated 24 ${ }^{\text {th }}$ February, 2014.

Consent to participate: This study was conducted using the biological samples collected from selected patients visited our OPD. For every patient, prior written consent was taken before enrolling him/her in the study.

Consent to publish: Not applicable

Acknowledgement:-The work was supported by National Tea Research Foundation, Govt. of India and DST-Inspire fellowship programme, Department of Science and Technology, Govt. of India, ICMR Research Associate Programme, Govt. of India.

\section{References}

1. Ahmadiany M, Alavi-Samani M, Hashemi Z et al (2019) The Increased RNase Activity of IRE1alpha in PBMCs from Patients with Rheumatoid Arthritis. Adv Pharm Bull 9:505-509. https://doi.org/10.15171/apb.2019.060

2. Aletaha D, Neogi T, Silman AJ et al (2010) 2010 rheumatoid arthritis classification criteria: an American College of Rheumatology/European League Against Rheumatism collaborative initiative. Ann Rheum Dis 69:1580-1588. https://doi.org/10.1136/ard.2010.138461

3. Alunno A, Carubbi F, Giacomelli R, Gerli R (2017) Cytokines in the pathogenesis of rheumatoid arthritis: new players and therapeutic targets. BMC Rheumatol 1:3. https://doi.org/10.1186/s41927017-0001-8

4. Andersson AM, Andersson B, Lorell C et al (2016) Autophagy induction targeting mTORC1 enhances Mycobacterium tuberculosis replication in HIV co-infected human macrophages. Sci Rep 6:28171. https://doi.org/10.1038/srep28171

5. Bello AE, Perkins EL, Jay R, Efthimiou P (2017) Recommendations for optimizing methotrexate treatment for patients with rheumatoid arthritis. Open Access Rheumatol 9:67-79. https://doi.org/10.2147/OARRR.S131668 
6. Bergstrom B, Carlsten H, Ekwall AH (2018) Methotrexate inhibits effects of platelet-derived growth factor and interleukin-1beta on rheumatoid arthritis fibroblast-like synoviocytes. Arthritis Res Ther 20:49. https://doi.org/10.1186/s13075-018-1554-7

7. Bustamante MF, Garcia-Carbonell R, Whisenant KD, Guma M (2017) Fibroblast-like synoviocyte metabolism in the pathogenesis of rheumatoid arthritis. Arthritis Res Ther 19:110. https://doi.org/10.1186/s13075-017-1303-3

8. Chun Y, Kim J (2018) Autophagy: An Essential Degradation Program for Cellular Homeostasis and Life. Cells 7:. https://doi.org/10.3390/cells7120278

9. Decuypere JP, Parys JB, Bultynck G (2012) Regulation of the autophagic bcl-2/beclin 1 interaction. Cells 1:284-312. https://doi.org/10.3390/cells1030284

10. Elshabrawy HA, Chen Z, Volin MV et al (2015) The pathogenic role of angiogenesis in rheumatoid arthritis. Angiogenesis 18:433-448. https://doi.org/10.1007/s10456-015-9477-2

11. Fairbanks LD, Ruckemann K, Qiu Y et al (1999) Methotrexate inhibits the first committed step of purine biosynthesis in mitogen-stimulated human T-lymphocytes: a metabolic basis for efficacy in rheumatoid arthritis? Biochem J 342(Pt 1):143-152

12. Gao Y, Rankin GO, Tu Y, Chen YC (2016) Theaflavin-3, 3'-digallate decreases human ovarian carcinoma OVCAR-3 cell-induced angiogenesis via Akt and Notch-1 pathways, not via MAPK pathways. Int J Oncol 48:281-292. https://doi.org/10.3892/ijo.2015.3257

13. Goncharova SA, Frankfurt OS (1976) Effect of methotrexate on the cell cycle of L1210 leukemia. Cell Tissue Kinet 9:333-340. https://doi.org/10.1111/j.1365-2184.1976.tb01281.x

14. Gosslau A, Li S, Zachariah E, Ho C-T (2018) Therapeutic Connection Between Black Tea Theaflavins and Their Benzotropolone Core Structure. Curr Pharmacol Reports 4:447-452. https://doi.org/10.1007/s40495-018-0157-y

15. Guo Q, Wang Y, Xu D et al (2018) Rheumatoid arthritis: pathological mechanisms and modern pharmacologic therapies. Bone Res 6:15. https://doi.org/10.1038/s41413-018-0016-9

16. Guzel E, Arlier S, Guzeloglu-Kayisli $O$ et al (2017) Endoplasmic Reticulum Stress and Homeostasis in Reproductive Physiology and Pathology. Int J Mol Sci 18:. https://doi.org/10.3390/ijms18040792

17. Herman S, Zurgil N, Deutsch M (2005) Low dose methotrexate induces apoptosis with reactive oxygen species involvement in T lymphocytic cell lines to a greater extent than in monocytic lines. Inflamm Res 54:273-280. https://doi.org/10.1007/s00011-005-1355-8

18. Heymann D (2006) Autophagy: A protective mechanism in response to stress and inflammation. Curr Opin Investig Drugs 7:443-450

19. Hu H, Tian M, Ding C, Yu S (2018) The C/EBP Homologous Protein (CHOP) Transcription Factor Functions in Endoplasmic Reticulum Stress-Induced Apoptosis and Microbial Infection. Front Immunol 9:3083. https://doi.org/10.3389/fimmu.2018.03083

20. Huang R, Liu W (2015) Identifying an essential role of nuclear LC3 for autophagy. Autophagy 11:852-853. https://doi.org/10.1080/15548627.2015.1038016 
21. Jung S, Jeong H, Yu SW (2020) Autophagy as a decisive process for cell death. Exp Mol Med 52:921-930. https://doi.org/10.1038/s12276-020-0455-4

22. Junjappa RP, Patil P, Bhattarai KR et al (2018) IRE1a Implications in Endoplasmic Reticulum StressMediated Development and Pathogenesis of Autoimmune Diseases. Front Immunol 9:1289. https://doi.org/10.3389/fimmu.2018.01289

23. Kabala PA, Angiolilli C, Yeremenko N et al (2017) Endoplasmic reticulum stress cooperates with Tolllike receptor ligation in driving activation of rheumatoid arthritis fibroblast-like synoviocytes. Arthritis Res Ther 19:207. https://doi.org/10.1186/s13075-017-1386-x

24. Kang EH, Kim DJ, Lee EY et al (2009) Downregulation of heat shock protein 70 protects rheumatoid arthritis fibroblast-like synoviocytes from nitric oxide-induced apoptosis. Arthritis Res Ther 11:R130. https://doi.org/10.1186/ar2797

25. Kato M, Ospelt C, Gay RE et al (2014) Dual role of autophagy in stress-induced cell death in rheumatoid arthritis synovial fibroblasts. Arthritis Rheumatol 66:40-48. https://doi.org/10.1002/art.38190

26. Khan N, Mukhtar H (2013) Tea and health: studies in humans. Curr Pharm Des 19:6141-6147. https://doi.org/10.2174/1381612811319340008

27. Krock BL, Skuli N, Simon MC (2011) Hypoxia-induced angiogenesis: good and evil. Genes Cancer 2:1117-1133. https://doi.org/10.1177/1947601911423654

28. Leone M, Zhai D, Sareth $S$ et al (2003) Cancer prevention by tea polyphenols is linked to their direct inhibition of antiapoptotic Bcl-2-family proteins. Cancer Res 63:8118-8121

29. Liu W, Li J (2019) Theaflavin-3, 3'-Digallate Attenuates Rheumatoid Inflammation in Mice Through the Nuclear Factor-kappaB and MAPK Pathways. Arch Immunol Ther Exp 67:153-160. https://doi.org/10.1007/s00005-019-00536-7

30. Luo X, Zuo X, Zhou Y et al (2008) Extracellular heat shock protein 70 inhibits tumour necrosis factoralpha induced proinflammatory mediator production in fibroblast-like synoviocytes. Arthritis Res Ther 10:R41. https://doi.org/10.1186/ar2399

31. MacDonald IJ, Liu SC, Su CM et al (2018) Implications of Angiogenesis Involvement in Arthritis. Int J Mol Sci 19:. https://doi.org/10.3390/ijms19072012

32. Nygaard G, Firestein GS (2020) Restoring synovial homeostasis in rheumatoid arthritis by targeting fibroblast-like synoviocytes. Nat Rev Rheumatol 16:316-333. https://doi.org/10.1038/s41584-0200413-5

33. Pan H, Li J, Rankin GO et al (2018) Synergistic effect of black tea polyphenol, theaflavin-3,3'-digallate with cisplatin against cisplatin resistant human ovarian cancer cells. J Funct Foods 46:1-11. https://doi.org/10.1016/j.jff.2018.04.037

34. Park YJ, Yoo SA, Kim WU (2014) Role of endoplasmic reticulum stress in rheumatoid arthritis pathogenesis. J Korean Med Sci 29:2-11. https://doi.org/10.3346/jkms.2014.29.1.2

35. Pincus T, Yazici Y, Sokka T et al (2003) Methotrexate as the "anchor drug" for the treatment of early rheumatoid arthritis. Clin Exp Rheumatol 21:S179-S185 
36. Qu X, Tang Y, Hua S (2018) Immunological Approaches Towards Cancer and Inflammation: A Cross Talk. Front Immunol 9:563. https://doi.org/10.3389/fimmu.2018.00563

37. Quinonez-Flores CM, Gonzalez-Chavez SA, Pacheco-Tena C (2016) Hypoxia and its implications in rheumatoid arthritis. J Biomed Sci 23:62. https://doi.org/10.1186/s12929-016-0281-0

38. Runwal G, Stamatakou E, Siddiqi FH et al (2019) LC3-positive structures are prominent in autophagydeficient cells. Sci Rep 9:10147. https://doi.org/10.1038/s41598-019-46657-z

39. Sakura T, Hayakawa F, Sugiura I et al (2018) High-dose methotrexate therapy significantly improved survival of adult acute lymphoblastic leukemia: a phase III study by JALSG. Leukemia 32:626-632. https://doi.org/10.1038/leu.2017.283

40. Sen R, Bandyopadhyay S, Dutta A et al (2007) Artemisinin triggers induction of cell-cycle arrest and apoptosis in Leishmania donovani promastigotes. J Med Microbiol 56:1213-1218. https://doi.org/10.1099/jmm.0.47364-0

41. Shen Z, Chen Q, Jin T et al (2019) Theaflavin 3,3'-digallate reverses the downregulation of connexin 43 and autophagy induced by high glucose via AMPK activation in cardiomyocytes. J Cell Physiol 234:17999-18016. https://doi.org/10.1002/jcp.28432

42. Smith MD, Walker JG (2004) Apoptosis a relevant therapeutic target in rheumatoid arthritis? Rheumatol 43:405-407. https://doi.org/10.1093/rheumatology/keh084

43. Stebulis JA, Rossetti RG, Atez FJ, Zurier RB (2005) Fibroblast-like synovial cells derived from synovial fluid. J Rheumatol 32:301-306

44. Steinsson K, Weinstein A, Korn J, Abeles M (1982) Low dose methotrexate in rheumatoid arthritis. J Rheumatol 9:860-866

45. Tu Y, Kim E, Gao Y et al (2016) Theaflavin-3, 3'-digallate induces apoptosis and G2 cell cycle arrest through the Akt/MDM2/p53 pathway in cisplatin-resistant ovarian cancer A2780/CP70 cells. Int J Oncol 48:2657-2665. https://doi.org/10.3892/ijo.2016.3472

46. Vomero M, Barbati C, Colasanti T et al (2018) Autophagy and Rheumatoid Arthritis: Current Knowledges and Future Perspectives. Front Immunol 9:1577. https://doi.org/10.3389/fimmu.2018.01577

47. Wang M, Wey S, Zhang Y et al (2009) Role of the unfolded protein response regulator GRP78/BiP in development, cancer, and neurological disorders. Antioxid Redox Signal 11:2307-2316. https://doi.org/10.1089/ARS.2009.2485

48. Wang W, Zhou H, Liu L (2018) Side effects of methotrexate therapy for rheumatoid arthritis: A systematic review. Eur J Med Chem 158:502-516. https://doi.org/10.1016/j.ejmech.2018.09.027

49. Wu R, Zhang QH, Lu YJ et al (2015) Involvement of the IRE1a-XBP1 pathway and XBP1s-dependent transcriptional reprogramming in metabolic diseases. DNA Cell Biol 34:6-18. https://doi.org/10.1089/dna.2014.2552

50. Xu K, Cai YS, Lu SM et al (2015) Autophagy induction contributes to the resistance to methotrexate treatment in rheumatoid arthritis fibroblast-like synovial cells through high mobility group box chromosomal protein 1. Arthritis Res Ther 17:374. https://doi.org/10.1186/s13075-015-0892-y 
51. Yang Y, Liu L, Naik I et al (2017) Transcription Factor C/EBP Homologous Protein in Health and Diseases. Front Immunol 8:1612. https://doi.org/10.3389/fimmu.2017.01612

52. Yoo SA, You S, Yoon HJ et al (2012) A novel pathogenic role of the ER chaperone GRP78/BiP in rheumatoid arthritis. J Exp Med 209:871-886. https://doi.org/10.1084/jem.20111783

53. Yu MB, Firek A, Langridge WHR (2018) Predicting methotrexate resistance in rheumatoid arthritis patients. Inflammopharmacology 26:699-708. https://doi.org/10.1007/s10787-018-0459-z

54. Zeng L, Zampetaki A, Margariti A et al (2009) Sustained activation of XBP1 splicing leads to endothelial apoptosis and atherosclerosis development in response to disturbed flow. Proc Natl Acad Sci U S A 106:8326-8331. https://doi.org/10.1073/pnas.0903197106

55. Zhu L, Wang H, Wu Y et al (2017) The Autophagy Level Is Increased in the Synovial Tissues of Patients with Active Rheumatoid Arthritis and Is Correlated with Disease Severity. Mediators Inflamm 2017:7623145. https://doi.org/10.1155/2017/7623145

\section{Table}

Due to technical limitations, the table is only available as a download in the supplemental files section.

\section{Figures}
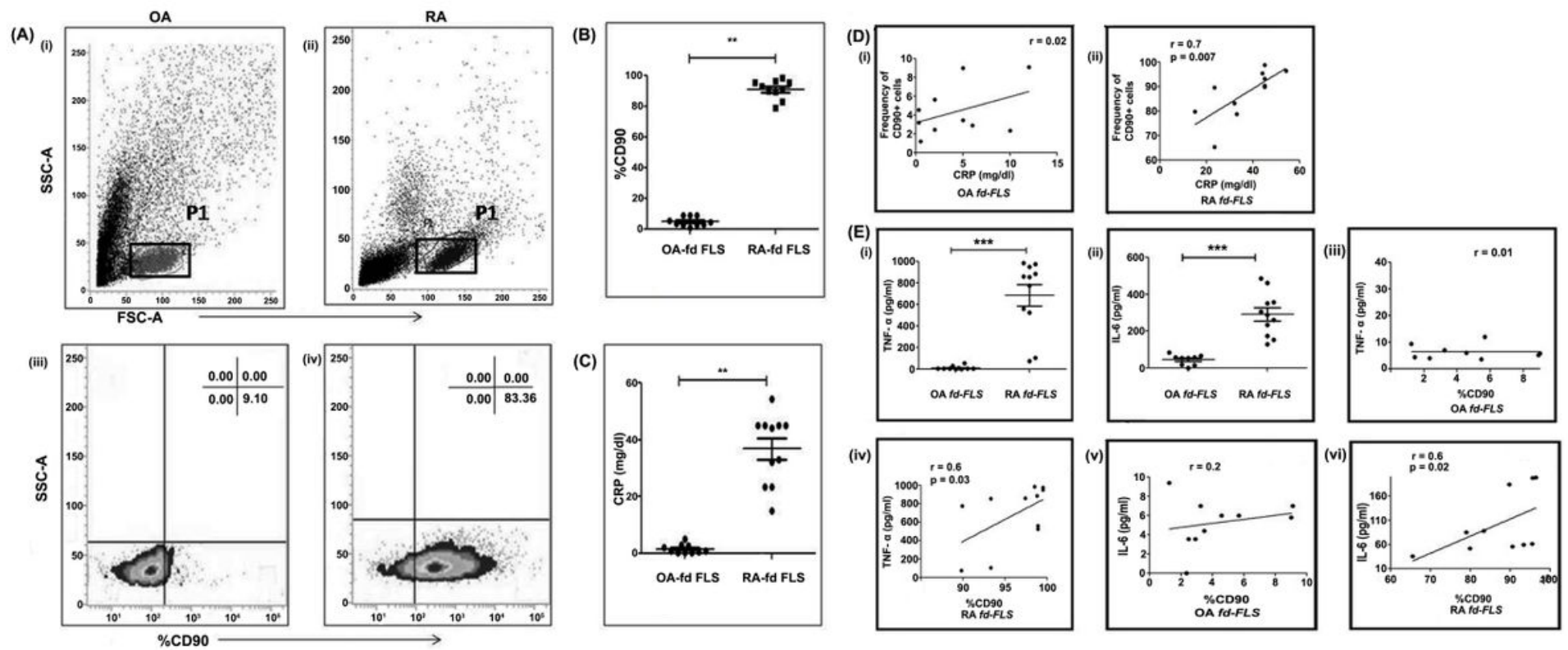

\section{Figure 1}

Assessment of inflammatory markers from cell soup of $\mathrm{fd}$-FLS. A) Characterization of fdFLS from synovial fluid using specific antibody (CD90) by FACS analysis. (i) Representative profile of synovial fibroblast (fd-FLS) gated P1 sourced from synovial fluid of OA patients. (ii) Representative profile of synovial fibroblast (fd-FLS) gated P1sourced from synovial fluid of a RA patients. (iii) Representative 
quadrant plot demonstrating frequency of CD90 positive in cultured OA synovial fluid. (iv) Representation quadrant plot demonstrating frequency of CD90 positive in cultured RA synovial fluid. B) Percentage of CD90+ cells from synovial fluid obtained from RA and OA. Data is indicated in median (IQR), ${ }^{\star *} \mathrm{p}<0.01$ as compared to OA fd-FLS. C) Estimation of CRP level from cultured fd-FLS of OA and RA after $24 \mathrm{~h}$ of incubation by ELISA technique. Data represents in median (IQR), ${ }^{\star *} \mathrm{p}<0.01$ as compared to OA fd-FLS. D) Inter-relationship between fd-FLS obtained from both RA and OA patients with inflammatory marker (i) Correlation of CD90+ cells and CRP in OA fd-FLS. (ii) Correlation between CD90+ cells and CRP in RA fdFLS. E) Pro-inflammatory cytokine level in OA fd-FLS and RA fd-FLS. (i) Expression of TNF-a between RA fd-FLS and OA fd-FLS. ${ }^{* \star} \times p<0.01$ as compared to OA fd-FLS. (ii) Expression of IL-6 between RA fd-FLS and OA fd-FLS. ${ }^{* * *} p<0.01$ as compared to OA fd-FLS. (iii) Correlation between TNF-a and CD 90 (Thy-1) in OA fd-FLS. (iv) Correlation between TNF-a and CD90 (Thy-1) in RA fd-FLS. (v) Correlation between IL-6 and CD90 (Thy-1) in OA fd-FLS. (vi) Correlation between IL-6 and CD90 (Thy-1) in RA fd-FLS.
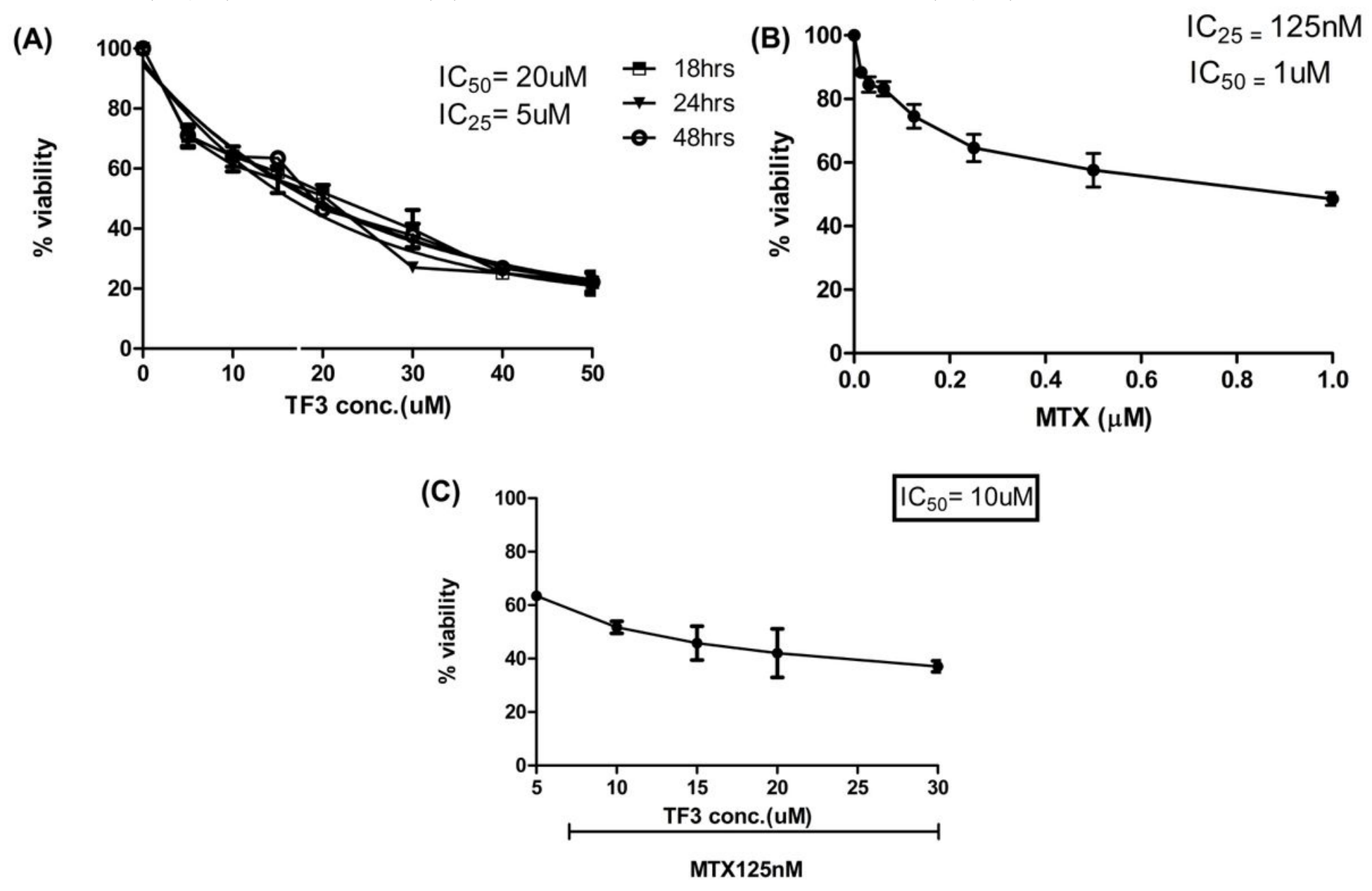

Figure 2

Cell viability assay after treatment with MTX and TF3 to fd-FLS from RA patients by MTT assay. A) The synergistic inhibitory effect of TF3 on cells was determined by MTT assay. Briefly, cultured cells (1X104) were treated with TF3 (0-50uM) for period of $(18,24,48 \mathrm{~h})$. B) Effect of MTX on fd-FLS from RA patients.1X104 cells/well was seeded on 96 well plates and were incubated for $24 \mathrm{~h}$ after treatment with 


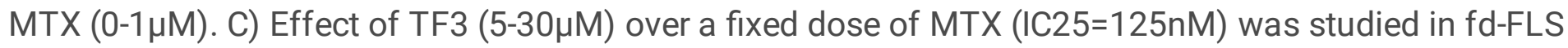
after incubation for $24 \mathrm{~h}$ followed by MTT assay.

(A)

$\mathrm{kDa}$

120

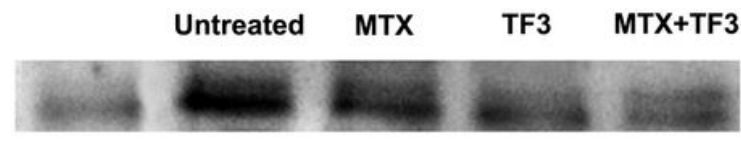

52

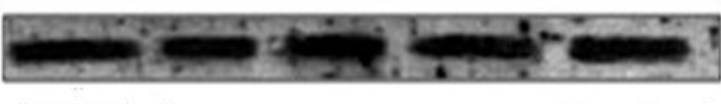

OA $f d-F L S$

RA $f d-F L S$

(C)

(i)

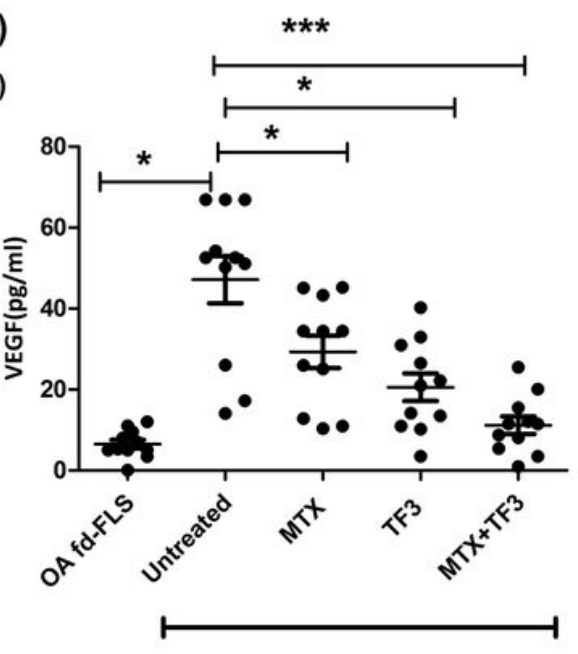

RA $f d-F L S$
HIF-1 $\alpha$

a Tubulin

(B)

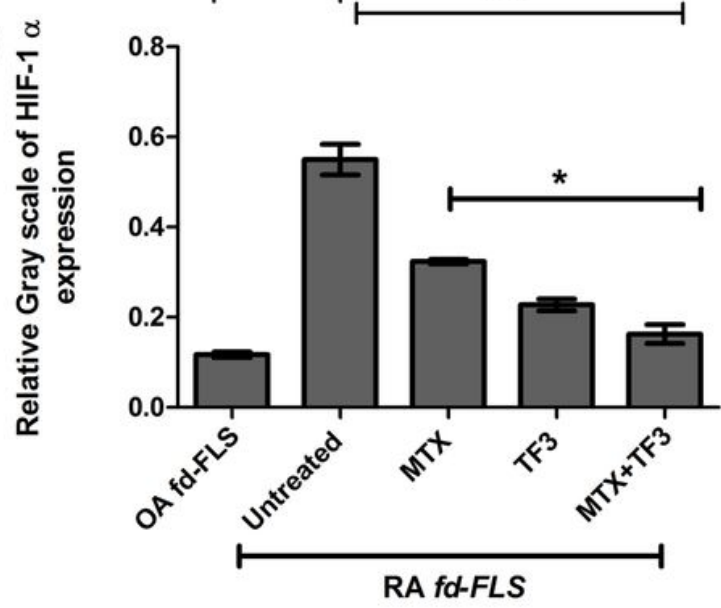

(ii)

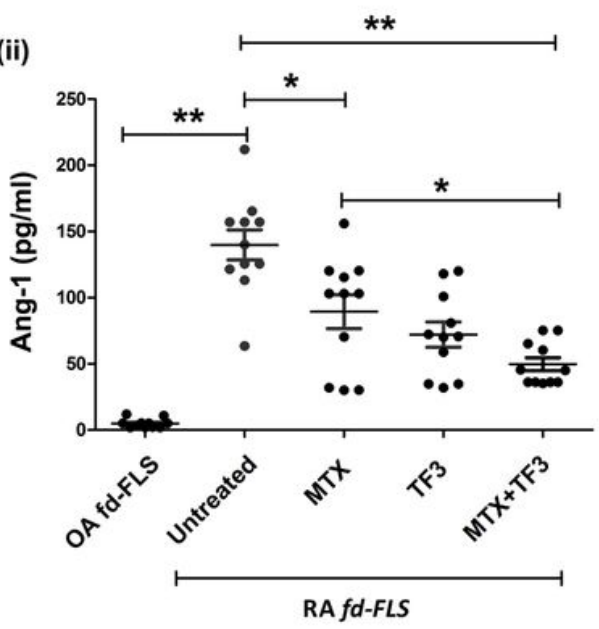

Figure 3

Determination of (HIF-1a) expression in fd-FLS after treatment with TF3 alone or in combination with MTX. A) Interpretation of HIF-1 a expression by immunoblot assay. B) Different experimental sets by densitometric scans of HIF-1a immunoblot. C) Determination of angiogenic markers. (i) Expression of VEGF, ${ }^{\star *} p \mathrm{p}<0.001$ compared to untreated group vs MTX+TF3. (ii) Expression of ANG-1, ${ }^{\star \star} p<0.01$ compared to untreated group vs MTX+TF3. 

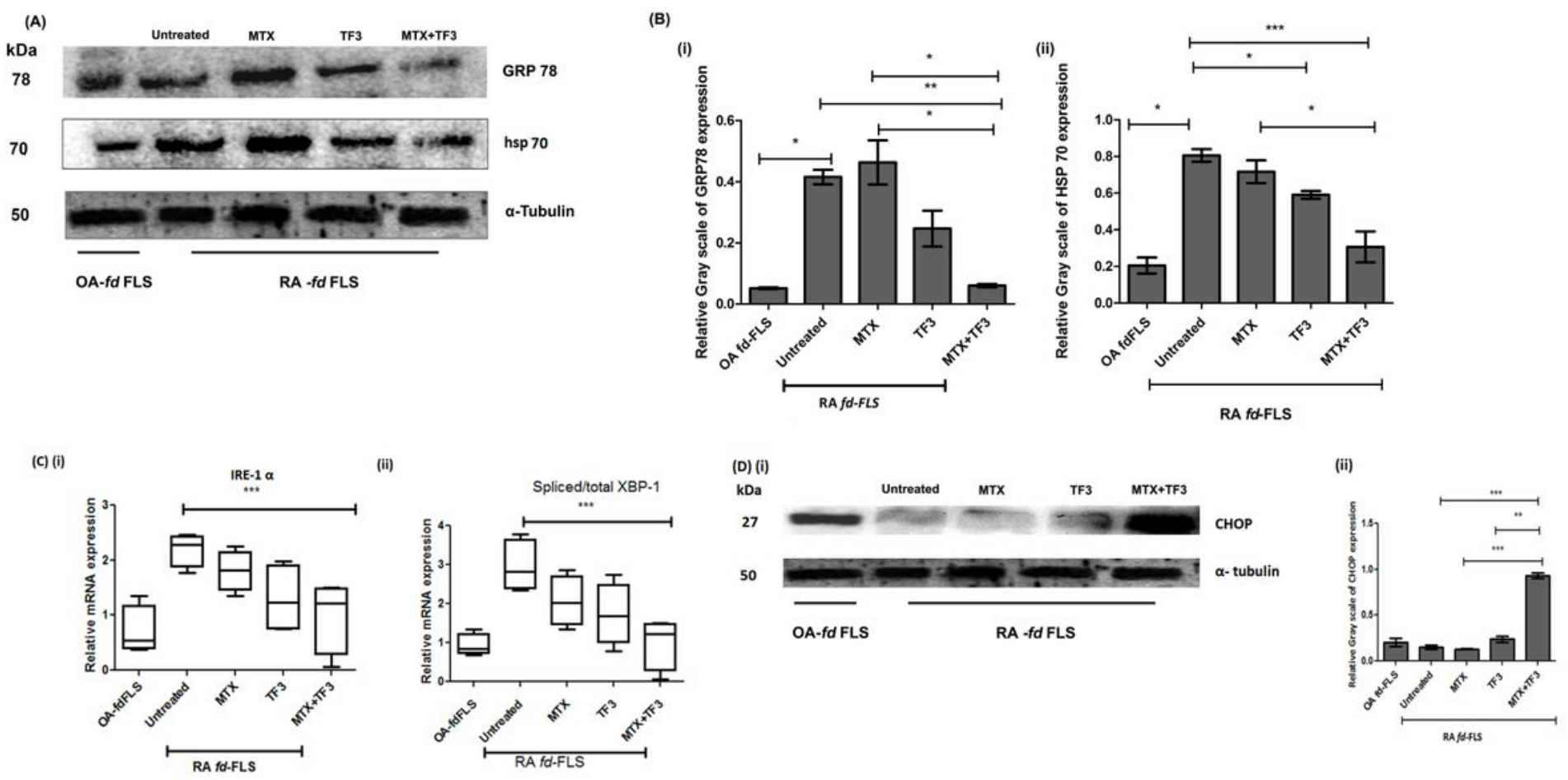

\section{Figure 4}

Determination of hypoxia mediated ER stress in fd-FLS from RA patients after combination treatment with TF3 and MTX. A) Expressions of GRP78 and hsp70 were determined by immunoblot technique. Data were normalized by a-tubulin. B) Immunoreactive bands were identified by densitometric scans in different studied groups. (i) GRP78 expression, ${ }^{*} p<0.05$ compared to untreated group vs MTX+TF3. (ii) HSP70 expression, ${ }^{\star} p<0.05$ compared to untreated group vs MTX+TF3. C) Expression of ER stress genes in RA patients. (i) Gene expression level of IRE-1a, (ii) sXBP-1. D) (i) CHOP expression determined by immunoblot assay. (ii) Densitometry scan of CHOP in different studied group. ${ }^{\star \star *} p<0.001$ compared to untreated group vs MTX+TF3. 

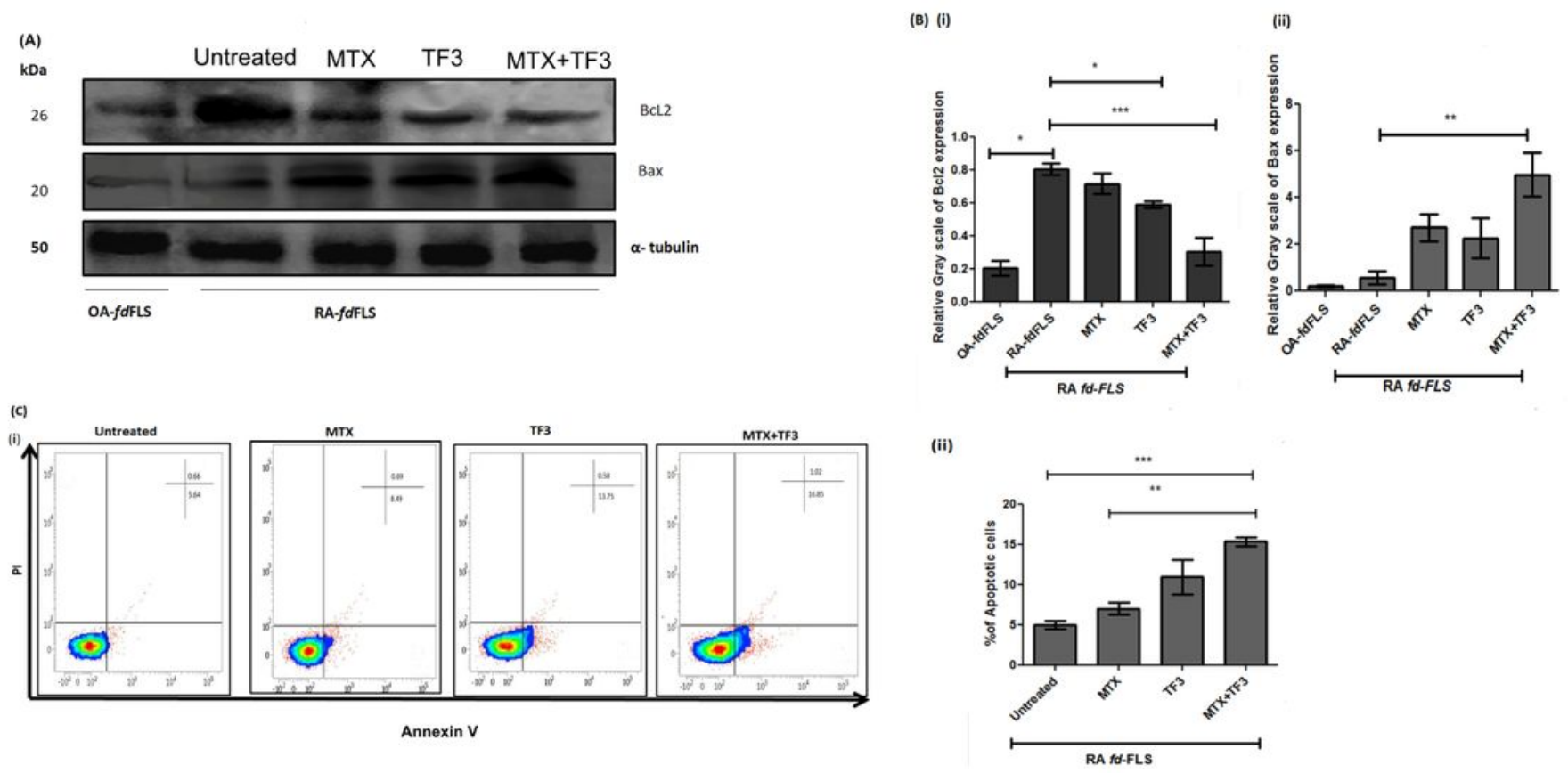

(D)
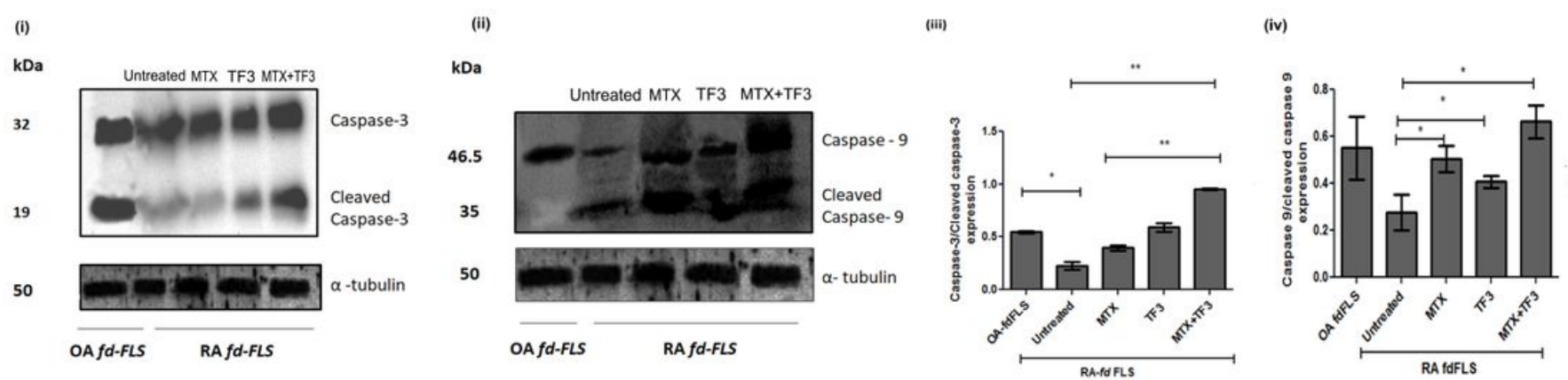

(E)

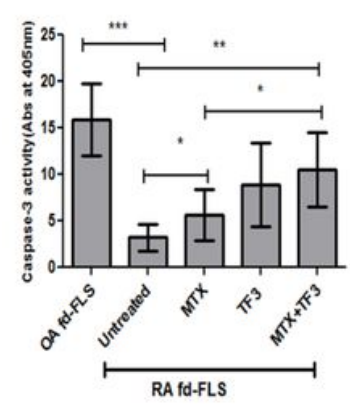

Figure 5

MTX+TF3 administration induced apoptosis in fd-FLS from RA patients. A) Representation of immunoblot data of $\mathrm{Bcl} 2$ and Bax after treatment with TF3, MTX and in combination. B) Densitometry scan of (i) Bcl2 (ii) Bax. C) (i) Cell death was measured by FACS analysis using Annexin V-FITC in fd-FLS after treatment with TF3 and MTX in combination. (ii) Percentage of Annexin V positive cells were represented in histogram. D) Immunoblot data after treatment with TF3, MTX and in combination. (i) Determination of caspase 3 and cleaved caspase 3. (ii) Expression of caspase 9 and cleaved caspase 9. Densitometry scans of (iii) Caspase-3 (iv) Caspase 9 in different studied groups. (E) Estimation of 
caspase 3 activity by colorimetric study in fd-FLS from RA after different drug administration. Data represent as mean $\pm S D\left({ }^{*} p<0.05,{ }^{*} p<0.01,{ }^{* *} p<0.001\right)$.

(A)
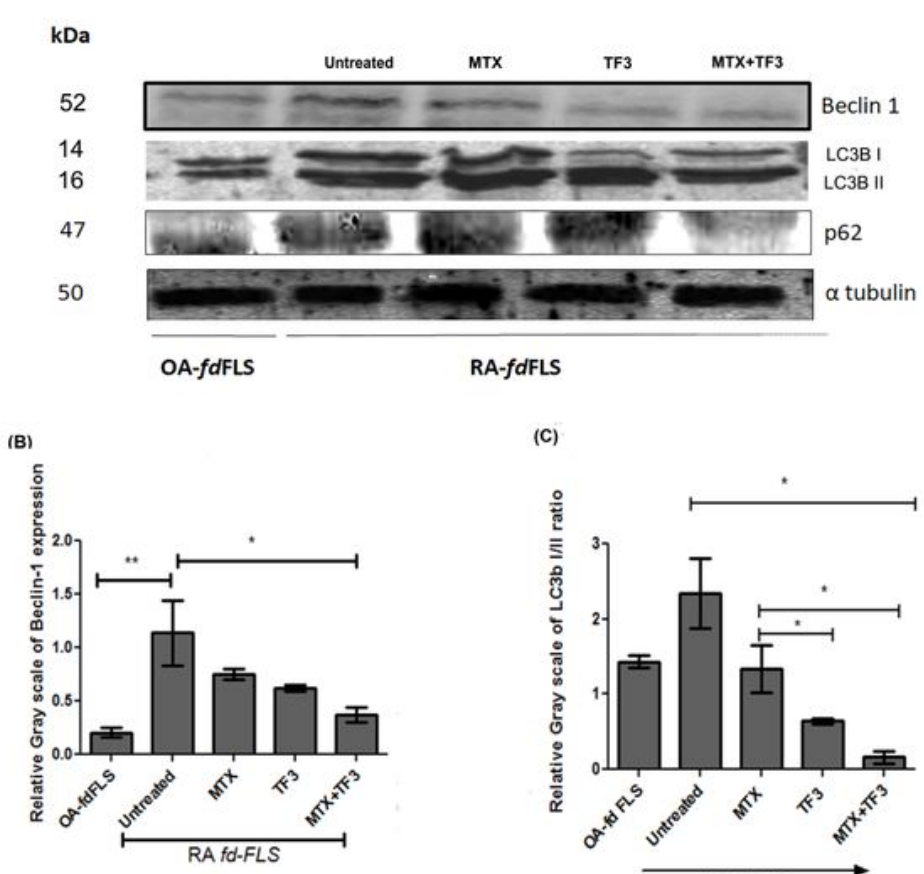

(C)

(D)

(E)
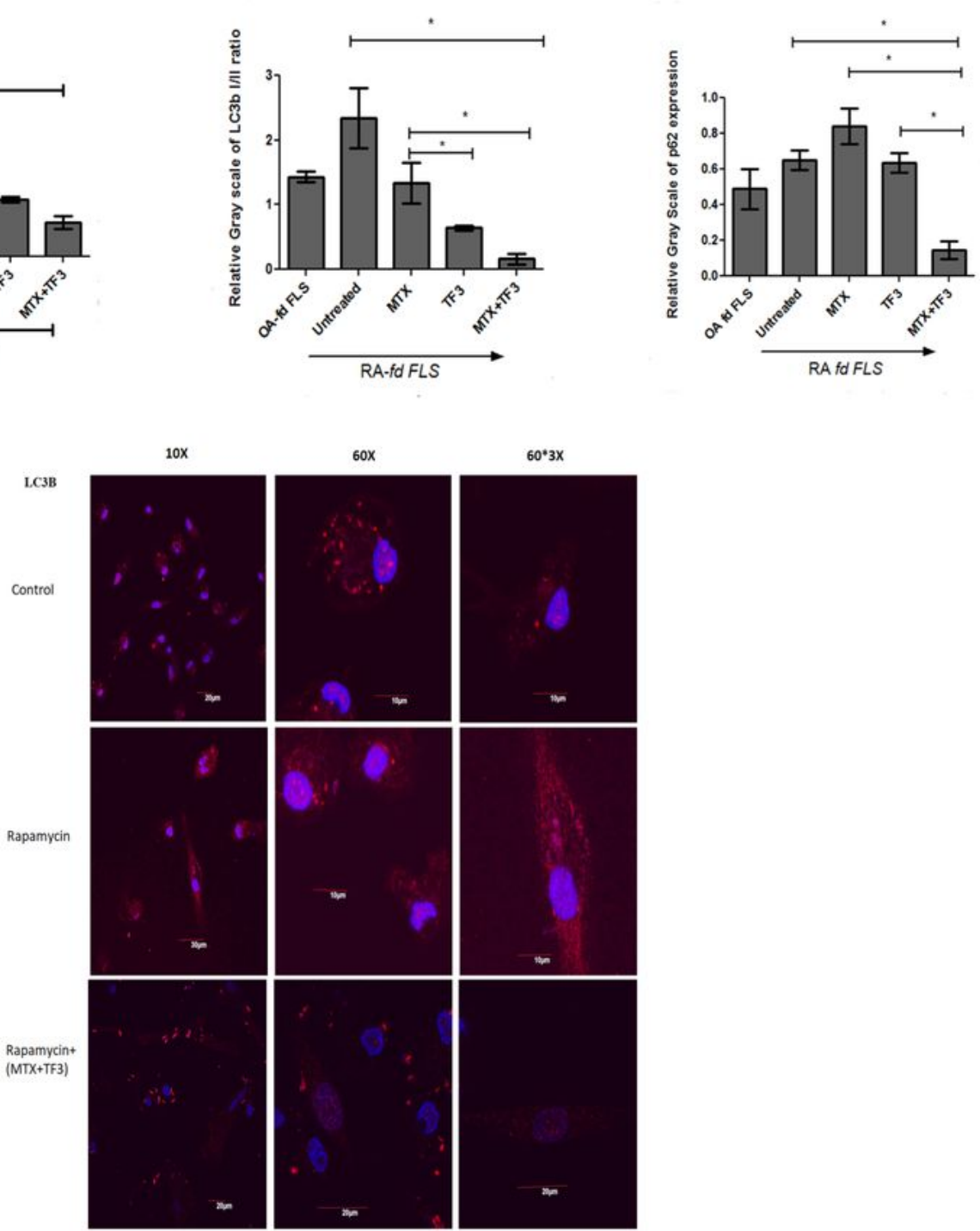

Figure 6

Determination of autophagic protein expression in fd-FLS from RA after combination treatment. A) Expression of Beclin-1, LC3bl, LC3bll and p62 by immunoblot studies. B) Densitometry scan of Beclin-1 expression. (C) Ratio of immunoreactive bands between LC3bl:LC3bll in different studied groups. D) 
Assessment of relative gray scale value of p62 protein from fd-FLS in different experimental group. E) Confocal image to demonstration the expression of LC3B in fd-FLS from RA patients using autophagy inducer (Rapamycin). fd-FLS were treated with rapamycin(200nM) along with TF3 and MTX for $24 \mathrm{hr}$ and was subjected to confocal imaging as elaborated in methodology section. LC3B marker was determined using $60(X)$ and $60 X * 3$ in oil immersion lens. The immunocytochemical staining was done by anti-LC3B antibody and alexafluor 647 secondary antibody. Data represent as mean $\pm S D\left({ }^{\star} p<0.05,{ }^{\star \star} p<0.01\right.$, $* * p<0.001)$.

(A)

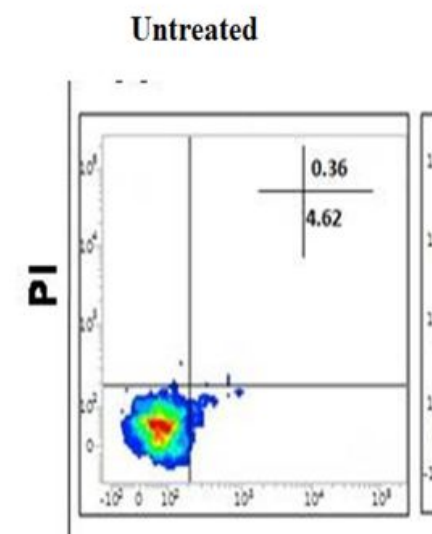

3MA

3MA+MTX

3MA+TF3

3MA+MTX+TF3
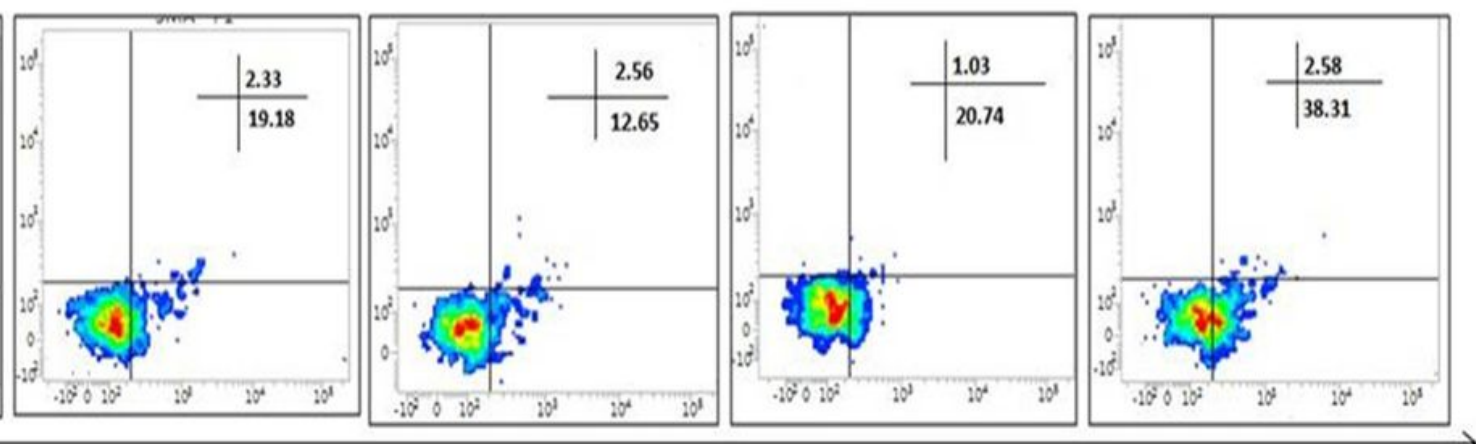

Annexin V

(B)

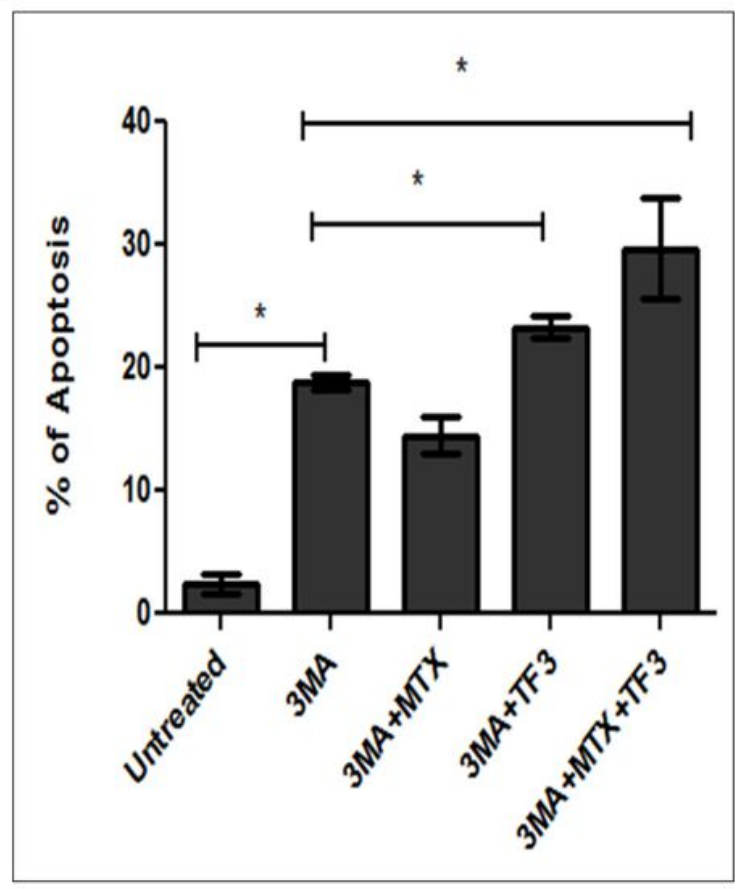

Figure 7

Effectiveness of combination therapy in fd-FLS from RA patients. A) fd-FLS were treated with autophagic blocker (3MA). Briefly cells (fd-FLS) were treated with $3 M A(10 \mathrm{nM})$ for $24 \mathrm{hr}$ along with MTX and TF3 after that apoptosis was estimated using Annexin V-FITC by FACS analysis. B) Determination of 
percentage of apoptotic cells in fd-FLS after 3-MA or combination therapy (MTX+TF3) Data represent as mean $\pm S D\left({ }^{*} p<0.05,{ }^{* *} p<0.01,{ }^{* *} p<0.001\right)$.

\section{Supplementary Files}

This is a list of supplementary files associated with this preprint. Click to download.

- Table1.ppt 